\title{
Modeling bacterial resistance to antibiotics: bacterial conjugation and drug effects
}

\author{
Pirommas Techitnutsarut ${ }^{1}$ and Farida Chamchod ${ }^{1,2^{*}}$
}

\author{
"Correspondence: \\ farida.cha@mahidol.ac.th \\ ${ }^{1}$ Department of Mathematics, \\ Faculty of Science, Mahidol \\ University, Rama VI, 10400 Bangkok, \\ Thailand \\ ${ }^{2}$ Center of Excellence in \\ Mathematics, Rama VI, 10400 \\ Bangkok, Thailand
}

\begin{abstract}
Antibiotic resistance is a major burden in many hospital settings as it drastically reduces the successful probability of treating bacterial infections. Generally, resistance is associated with bacterial fitness reduction and selection pressure from antibiotic usage. Here, we investigate the effects of bacterial conjugation, plasmid loss, and drug responses on the population dynamics of sensitive and resistant bacteria by using a mathematical model. Two types of drugs are considered here: antibiotic $\mathrm{M}$ that kills only sensitive bacteria and antibiotic $N$ that kills both bacteria. Our results highlight that larger dose and longer dosing interval of antibiotic $M$ may result in the higher prevalence of resistant bacteria while they do the opposite for antibiotic $\mathrm{N}$. When delays in administering initial and second doses are incorporated, the results demonstrate that the delays may lead to the higher prevalence of resistant bacteria when antibiotic $\mathrm{M}$ or $\mathrm{N}$ is administered with the longer time of bacteria remaining at the lower prevalence of the latter. Our results highlight that switching antibiotic agents during a treatment course and different bacterial strain characteristics result in a significant impact on the prevalence of resistant bacteria.
\end{abstract}

Keywords: Antimicrobial resistance; Bacterial conjugation; Therapeutic strategies; Mathematical modeling

\section{Introduction}

Antimicrobial-resistant bacteria (ARB) are a challenging and growing problem of public health worldwide as infections caused by them are harder to treat than those by nonresistant bacteria and may lead to longer hospital stay, additional costs, and increased mortality. Acquisition of ARB occurs in two different ways including mechanical emergence of resistance in an individual and transmission of bacteria already resistant to therapeutic agents among colonized individuals. With the extensive use and misuse of antimicrobials, the problem becomes worsened, and resistance to multiple drugs has been more frequently reported $[1,2]$. As ARB can also be transmitted via contact between patients and contaminated healthcare workers or environmental surfaces, the presence of them is widespread in many hospital settings and health-care facilities [3-5].

Generally, bacteria can resist the action of a particular antibiotic as a result of inherent structural and functional characteristics [6]. However, resistant genes can also be acquired via either mutations in chromosomal genes or horizontal gene transfer (HGT). Chromoso-

(c) The Author(s) 2021. This article is licensed under a Creative Commons Attribution 4.0 International License, which permits use, sharing, adaptation, distribution and reproduction in any medium or format, as long as you give appropriate credit to the original author(s) and the source, provide a link to the Creative Commons licence, and indicate if changes were made. The images or other third party material in this article are included in the article's Creative Commons licence, unless indicated otherwise in a credit line to the material. If material is not included in the article's Creative Commons licence and your intended use is not permitted by statutory regulation or exceeds the permitted use, you will need to obtain permission directly from the copyright holder. To view a copy of this licence, visit http://creativecommons.org/licenses/by/4.0/. 
mal mutation is the modification of DNA occurring at a low level during DNA replication $[7,8]$. Although spontaneous mutations can generate mutants that confer resistance to antibiotics, frequencies vary drastically according to bacterial species and therapeutic agents $[8,9]$. Horizontal gene transfer (HGT) is a process that allows microorganisms like bacteria to acquire genetic information from other microorganisms. HGT often involves the use of plasmids, transposons, and integrons [10]. There are three principal mechanisms of HGT including transformation, transduction, and conjugation. The latter has been recognized as a key mechanism for resistant gene dissemination in the hospital environment under antibiotic treatment $[10,11]$.

Mutations occurring from conjugation and chromosomal mutation can arise when bacteria are exposed to antibiotics at a sublethal concentration [12]. Such concentration may result from incomplete treatment, patient non-compliance, and limited drug accessibility to certain tissues [11]. It has been suggested that low level of antibiotics may induce mutagenesis in a wider range of antibacterial resistance genes [13]. Moreover, preceding studies suggest that horizontal evolution which is responsible for many current resistance problems occurs at high rates in the gastrointestinal tract of humans under antimicrobial therapy and causes resistance to spread from environmental species to pathogenic species $[14,15]$. Consequently, proper dose and duration of treatment are required to minimize the development of resistance.

A series of modeling studies has been conducted to help investigate the effects of antimicrobial therapies on the ascent of bacterial resistance. Some studies focused on how the immune response affects the dynamics of resistant bacteria when chromosomal mutation is present [16] and how it together with treatment timing can effectively help clear an infection [17]. In addition, in the presence of host immunity, Ternent et al. [18] demonstrated that combining antibiotics and anti-virulence drugs could help clear bacterial infections and prevent the emergence of antibiotic-resistant populations. By particularly taking into account bacterial conjugation, D'Agata et al. [19] showed that early initiation and combination of antibiotics could help prevent the emergence of resistant bacteria while shorter therapeutic courses and sequential administration of antibiotics did the opposite. However, rotation between different antibiotics was demonstrated to reduce the prevalence of antibiotic resistance in [20]. Several studies explored drug strategies to optimize antibiotic usage such as drug synergistic and antagonistic effects on the risk of multi-drug resistance, cycling between application and withdrawal of the antibiotic resulting in the fastest killing of bacteria, and a high initial dose followed by an extended tapering of doses optimising the use of antibiotics. [21-23]. There are also some preceding studies based on the pharmacokinetic and pharmacodynamic frameworks that explored the effects of pharmacodynamic parameters on the efficacy of antibiotics, optimal dosing regimens, multiple-drug therapy, or the minimum concentration that resistant bacteria are selected [24-28]. In this study, a mathematical model is developed by taking into account bacterial conjugation, probability of losing a plasmid, and antibiotic therapy. The model is implemented for identifying important factors that affect the prevalence of sensitive and resistant bacteria and investigating the effects of drug regimens, inhibitory types of antibiotics, delay of administration of the initial and second doses, antibiotic switching, and characteristics of bacterial strains. 


\section{Model formulation}

To investigate drug effects on the presence of antibiotic-resistant bacteria, a mathematical model based on a within-host framework is developed. In the compartmental model, we divide a population of bacteria into two categories: sensitive $(S)$ and $\operatorname{resistant}(R)$. Two processes including horizontal gene transfer and reversion of antibiotic resistance are taken into account in the model to describe interchangeability of bacteria between these two groups. Flow movements between the compartments are illustrated in Fig. 1. Parameters used in the model are depicted in Table 1. They are based on parameters for Staphylococcus aureus and some possible values. Population dynamics of sensitive and resistant bacteria are then given by

$$
\begin{aligned}
& \frac{d S}{d t}=g_{S}\left(1-\frac{S+R}{K}\right) S-\frac{\lambda S R}{S+R}+g_{R} \frac{p}{2} R-\left(\mu_{S}(A)+d\right) S, \\
& \frac{d R}{d t}=g_{R}\left(1-\frac{S+R}{K}\right) R+\frac{\lambda S R}{S+R}-g_{R} \frac{p}{2} R-\left(\mu_{R}(A)+d\right) R .
\end{aligned}
$$

This model is adapted from preceding studies by extending the models to explore therapeutic strategies $[16,19,34]$. In the model, growths of two populations of bacteria are described by the logistic terms with the shared carrying capacity $K$ and the division rates of sensitive and resistant bacteria, $g_{S}$ and $g_{R}$. Consequently, the total number of bacteria lies between 0 and $K$. Without drug effects, bacteria die naturally at a rate of $d$.
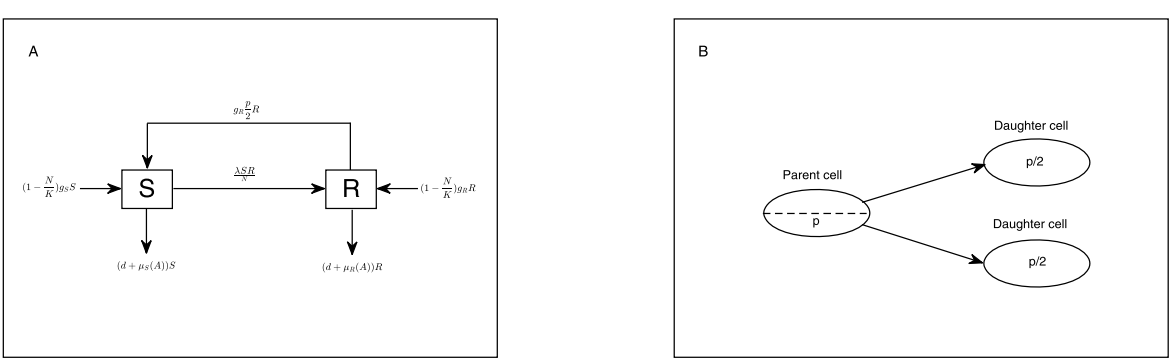

Figure 1 Model formulation. (a) A flow diagram for describing the population dynamics of sensitive $(S)$ and resistant $(R)$ bacteria, and (b) a binary fission diagram of a bacterial cell with the plasmid reversion probability

Table 1 Parameter values for the model

\begin{tabular}{lllll}
\hline Parameter & Description & Value & Units & Ref. \\
\hline$\lambda$ & Conjugation rate & 0.7 & $h^{-1}$ & from $R_{0}$ \\
$K$ & Bacteria carrying capacity & $10^{9}$ & Cells & {$[18]$} \\
$g_{S}$ & Growth rate of sensitive bacteria & 1 & $h^{-1}$ & {$[18,29]$} \\
$g_{R}$ & Growth rate of resistant bacteria & 0.65 & $h^{-1}$ & {$[18,29]$} \\
$p$ & Probability of losing a plasmid & 0.5 & - & varied \\
$d$ & Natural death rate & 0.5 & $h^{-1}$ & \\
$E_{\max }^{S}$ & Maximal kill rate of sensitive bacteria & 1.5 & $h^{-1}$ & {$[18,30]$} \\
$E_{\max }^{R}$ & Maximal kill rate of resistant bacteria & 1.1 & $h^{-1}$ & {$[18,30]$} \\
$A_{50}^{S}$ & Concentration of antibiotic which has half maximum effect & 0.25 & $\mu \mathrm{g} / \mathrm{ml}$ & {$[18,31]$} \\
$A_{50}^{R}$ & On sensitive bacteria & & & \\
& Concentration of antibiotic which has half maximum effect & 5 & $\mu \mathrm{g} / \mathrm{ml}$ & {$[18,31]$} \\
$\alpha_{A}$ & On resistant bacteria & & \multicolumn{2}{c}{} \\
\hline & Elimination rate of antibiotic under an intravenous therapy & 0.15 & $h^{-1}$ & {$[18,32,33]$} \\
\hline
\end{tabular}


Due to the ability to exchange genetic material of bacteria by the horizontal gene transfer process, it is assumed in the present study that acquisition of resistance commonly occurs via conjugation [14] so that sensitive bacteria become resistant ones after exchanging plasmids or transposons with resistant bacteria. We assume that conjugation is frequencydependent so that it does not depend on the bacterial population and it occurs at a rate of $\lambda$ with the probability of resistance acquisition $R /(S+R)$.

In contrast to acquisition of resistance, plasmid-bearing or resistant bacteria can become plasmid-free or sensitive bacteria from the bacteria reversion process $[8,35]$. In the above model, it is assumed that such a process happens at a rate of $g_{R} \frac{p}{2}$, where $p$ is the probability that a bacteria cell loses its resistant element during division. Note that during the binary fission, a plasmid-bearing bacterium generates two daughter cells so that the probability of losing a plasmid $p$ in the parent cell becomes $\frac{p}{2}$ in each daughter cell (see Fig. 1(b)).

To take into account the ability of an antibiotic agent to kill bacteria, we incorporate the drug killing rate as a sigmoid $E_{\max }$ function in model (1) as follows:

$$
\mu_{i}(A)=\frac{E_{\max }^{i} A(t)}{A_{50}^{i}+A(t)} \quad \text { for } i=S \text { or } R,
$$

where $E_{\max }^{i}$ is the maximum killing rate of bacteria $\mathrm{i}$, and $A_{50}^{i}$ represents the antibiotic concentration required for a half maximum effect [36]. In addition, $A(t)$ is the antibiotic concentration with an initial dose $A_{0}$ that satisfies the following equation:

$$
\frac{d A}{d t}=-\alpha A,
$$

where $\alpha$ denotes an antibiotic elimination rate. Hence, the antibiotic concentration exponentially diminishes over time $\left(A=A_{0} e^{-\alpha t}\right)$, and consequently the rate of killing decreases and approaches zero. In this work, two types of antibiotics are considered: 1 ) an antibiotic agent $M$ that kills only sensitive bacteria and 2) an antibiotic agent $\mathrm{N}$ that kills both bacteria. Moreover, owing to the ability of administered agents to normally kill more sensitive bacteria than resistant bacteria, we assume that $E_{\max }^{S} \geq E_{\max }^{R}$, and therefore $\mu_{S}(A) \geq \mu_{R}(A)$.

To study the effects from administrating multiple doses of antimicrobial agents during a treatment period, we implement a switch function that a constant dose of antibiotic is introduced at every $\tau$ period. Hence, the drug concentration becomes

$$
A_{i}(t)= \begin{cases}\left(\sum_{j=0}^{i-1} A_{0} e^{-j \alpha \tau}\right) e^{-\alpha(t-(i-1) \tau)}, & t \in((i-1) \tau, i \tau), \\ \sum_{j=0}^{i} A_{0} e^{-j \alpha \tau}, & t=i \tau,\end{cases}
$$

where $A_{i}$ is the antibiotic concentration after the $i$ th dose. This function reflects the possibility of having a remaining amount of drug from previous doses. Such a remaining amount depends on how fast the antibiotic agent is eliminated and how long from the beginning of the antibiotic course.

Since the total number of bacteria can be very large so that it may be difficult to compare the difference between sensitive and resistant bacteria, we rescale variables to study them in fractional numbers as follows:

$$
s=\frac{S}{K} \quad \text { and } \quad r=\frac{R}{K}
$$


Hence, system (1) becomes

$$
\begin{aligned}
& \frac{d s}{d t}=(1-(s+r)) g_{S} s-\frac{\lambda s r}{s+r}+g_{R} \frac{p}{2} r-\left(\mu_{S}(A)+d\right) s, \\
& \frac{d r}{d t}=(1-(s+r)) g_{R} r+\frac{\lambda s r}{s+r}-g_{R} \frac{p}{2} r-\left(\mu_{R}(A)+d\right) r .
\end{aligned}
$$

\section{Results}

\subsection{The resistance acquisition number $\left(R_{0}\right)$}

The resistance-free equilibrium of system (5) is given by $E_{0}=\left(1-\frac{d}{g_{S}}, 0\right)$. We calculate the resistance acquisition number $\left(R_{0}\right)$ that helps determine whether resistant bacteria can coexist with susceptible bacteria by using the next generation matrix method [37]. This number is similar to the basic reproduction number in epidemiology. If $R_{0}>1$, the resistant bacteria coexist with the sensitive bacteria; if $R_{0}<1$, only sensitive bacteria exist. Rearranging system (5) gives

$$
\frac{d X}{d t}=\mathcal{F}-\mathcal{V}
$$

where

$$
\begin{aligned}
& X=\left[\begin{array}{l}
r \\
s
\end{array}\right], \quad \mathcal{F}=\left[\begin{array}{c}
\frac{\lambda s r}{s+r} \\
0
\end{array}\right], \quad \text { and } \\
& \mathcal{V}=\left[\begin{array}{c}
-(1-(s+r)) g_{R} r+g_{R} \frac{p}{2} r+\left(\mu_{R}(A)+d\right) r \\
-(1-(s+r)) g_{R} s+\frac{\lambda s r}{s+r}-g_{R} \frac{p}{2} r+\left(\mu_{S}(A)+d\right) s
\end{array}\right] .
\end{aligned}
$$

Note that $\mathcal{F}$ is a vector of new acquisition of resistance, and $\mathcal{V}$ is a vector of other movements between the bacterial compartments. Next, we obtain $F$ and $V$ from the Jacobian matrices of $\mathcal{F}$ and $\mathcal{V}$ as shown below

$$
F=[\lambda], \quad \text { and } \quad V=\left[-g_{R}\left(1-\frac{p}{2}\right)+\frac{g_{R}}{g_{S}}\left(g_{S}-d\right)+d\right]
$$

Therefore, $R_{0}$ can be calculated as the spectral radius of $F V^{-1}$ and it is given by

$$
R_{0}=\frac{\lambda}{-g_{R_{A}}\left(1-\frac{p}{2}\right)+\frac{g_{R_{A}}}{g_{S}}\left(g_{S}-d\right)+d} .
$$

It can be clearly seen that based on the definition of resistance acquisition number, whether resistant bacteria persist depends on conjugation rate, growth rate, natural death rate, and probability of losing a plasmid. For example, high conjugation rate may lead to higher numbers of resistant bacteria.

\subsection{Numerical results}

To investigate an impact of certain parameters on the dynamics of sensitive and resistance bacteria in the model, we use numerical simulations based on the ODE45 function in MATLAB. In this work, $R_{0}$ is approximately 2 , which is assumed to be in a possible range of the resistance acquisition number of MRSA transmission between patients in some previous studies and also coincides with other antibiotic resistant bacteria [38, 39]. 

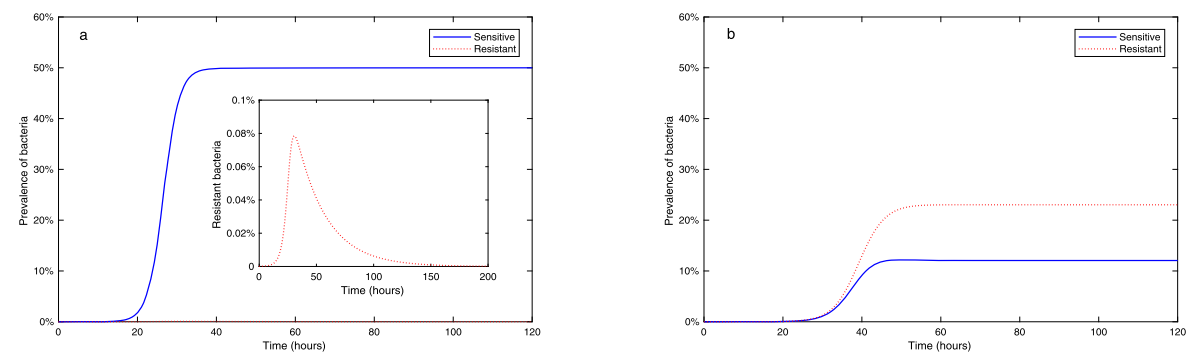

Figure 2 Prevalence of sensitive and resistant bacteria according to $R_{0}$ : (a) Resistant bacteria die out when $R_{0}<1$ $(\lambda=0.3)$, and (b) sensitive and resistant bacteria coexist when $R_{0}>1(\lambda=0.7)\left(S(0)=R(0)=10^{3}\right)$
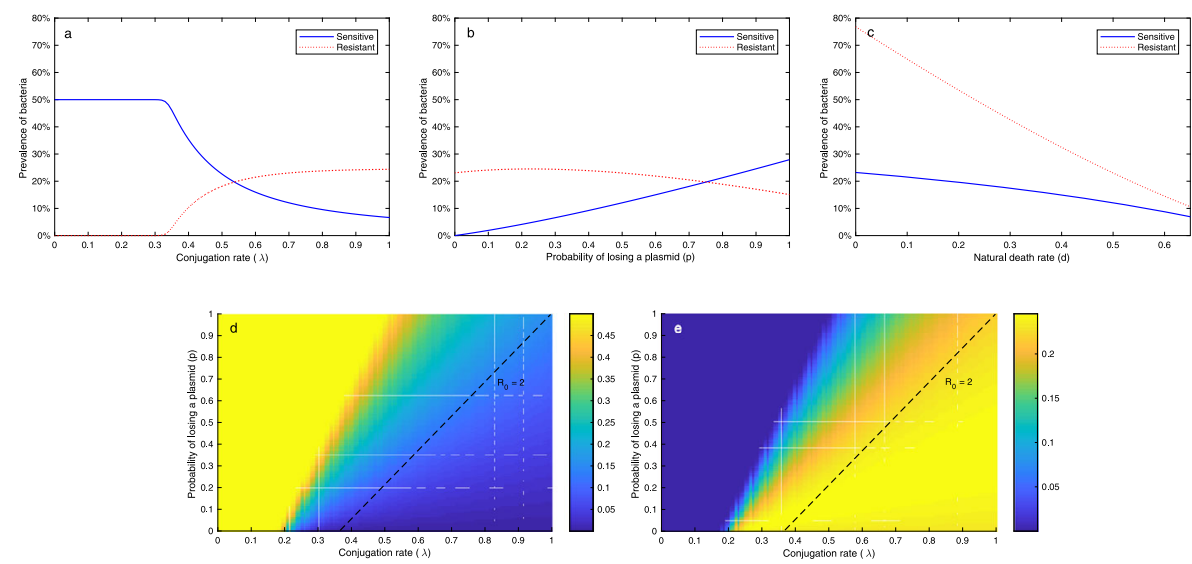

Figure 3 Effects of vital parameters on the prevalence of two bacterial types. Parameters, unless varied, are set according to Table 1. Results are obtained at the steady state when (a) the conjugation rate $\lambda$ is varied, (b) the probability of losing a plasmid $p$ is varied, and (c) the mortality rate of bacteria $d$ is varied. Prevalence of sensitive and resistant bacteria when both of the conjugation rate and the probability of losing a plasmid are varied is illustrated in $(\mathbf{d})$ and $(\mathbf{e})$, respectively $\left(S(0)=R(0)=10^{3}\right)$

First, we consider the system without antibiotic therapy $\left(\mu_{S}(A)=\mu_{R}(A)=0\right)$. Figure 2 illustrates that the resistance acquisition number helps determine whether the resistant bacteria population persists. In Fig. 2(a), resistant bacteria die out while sensitive bacteria persist when $R_{0}<1$. Both types of bacteria coexist whenever $R_{0}>1$ as shown in Fig. 2(b).

Figure 3(a) shows the effects of certain parameters on the prevalence of two bacteria at the steady states when bacterial conjugation is present. Based on our parameter setting, resistant bacteria are not prevalent until the conjugation rate approximately reaches 0.35 . Moreover, the system starts to favor resistant bacteria after the conjugation rate approximately approaches 0.55 . Our results generally suggest that the increasing conjugation rate favors the presence of resistant bacteria before the system is saturated. By varying the probability of losing a plasmid, it can be seen in Fig. 3(b) that the increased probability can eventually lead to a better outcome for sensitive bacteria. In this work, if the probability is roughly above 0.75 , sensitive bacteria become more prevalent than resistant bacteria. As the death rate is set to be equivalent for both sensitive and resistant, Fig. 3(c) shows an advantage of resistant bacteria over sensitive bacteria when the death rate is small. However, when the death rate becomes bigger, the prevalence of resistant bacteria is drastically reduced and becomes closer to the prevalence of sensitive bacteria. We further investigate 

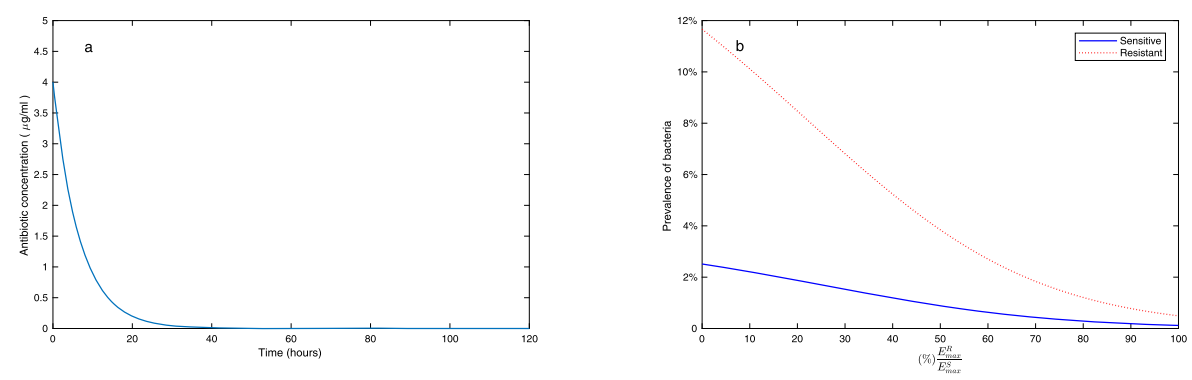

Figure 4 Drug concentration and efficacy. (a) Antibiotic concentration profile with the initial dose $A_{0}=4 \mu \mathrm{g} / \mathrm{ml}$, and $(\mathbf{b})$ the ratio between the maximum kill rate of resistant bacteria and the maximum kill rate of sensitive bacteria $\left(S(0)=R(0)=10^{3}\right)$

the effects of bacterial conjugation rate and the probability of losing a plasmid simultaneously in Fig. 3(d) \& (e). Our results suggest an impact from both the conjugation rate and the probability of losing a plasmid on the prevalence of sensitive and resistant bacteria with a lower impact from the latter. For example, our results suggest that the higher conjugation rate could lead to the higher prevalence of resistant bacteria but the lower prevalence of sensitive bacteria. However, the prevalence of resistant bacteria is slightly reduced when the probability of losing a plasmid becomes bigger. When the conjugation rate is small, the prevalence of sensitive bacteria is high while the prevalence of resistant bacteria is low.

Next, we incorporate administration of one dose of antibiotic into the model to investigate how drug effects influence the prevalence of sensitive and resistant bacteria. Figure 4(a) shows the decrease of drug concentration with the initial dose $A(0)=4 \mu \mathrm{g} / \mathrm{ml}$ and elimination rate $\alpha=0.15$ according to time. The concentration decreases rapidly within 20 hours and approaches 0 as time increases. In Fig. 4(b), we vary the ratio between the maximum kill rate of resistant bacteria and the maximum kill rate of sensitive bacteria in per cent (\% of $\frac{E_{\max }^{R}}{E_{\max }^{S}}$ ) so that $\frac{E_{\max }^{R}}{E_{\max }^{S}}=1$ means that the antibiotic acts equivalently to kill both types of bacteria. Our results show that resistant bacteria are dramatically reduced by the antibiotic efficacy to kill resistant bacteria. However, in comparison with the resistant bacteria, such an efficacy has a smaller impact on the sensitive bacterial population. In other words, the ability of antibiotic to kill resistant bacteria may not have a much negative impact on sensitive bacteria.

Figure 5(a) shows a profile of antibiotic concentration according to changes of the drug elimination rate at three different levels (slow, intermediate, fast). It can be clearly seen that a slower rate leads to a longer period of drug depletion. In Fig. 5(b)-(c), we demonstrate the behaviors of both populations of bacteria in correspondence with antibiotic agents $\mathrm{M}$ and $\mathrm{N}$, respectively. For the antibiotic agent $\mathrm{M}$, as it only affects the sensitive bacteria, the prevalence of sensitive bacteria decreases during the therapeutic effect ( $\sim 15 \mathrm{hrs}$ ), while the prevalence of resistant bacteria decreases but remains at a higher level than the sensitive bacteria. The latter event may result from the diminished conjugation pathway towards resistance following lower abundance of sensitive bacteria. Moreover, since it takes longer for the antibiotic agent to diminish, the sensitive population increases more slowly when the elimination rate decreases (see Fig. 5(b)). In case the antibiotic agent $\mathrm{N}$ that affects both populations of bacteria is applied, both bacterial populations decrease during the presence of therapeutic effects and then increase again after the drug clearance. In a 


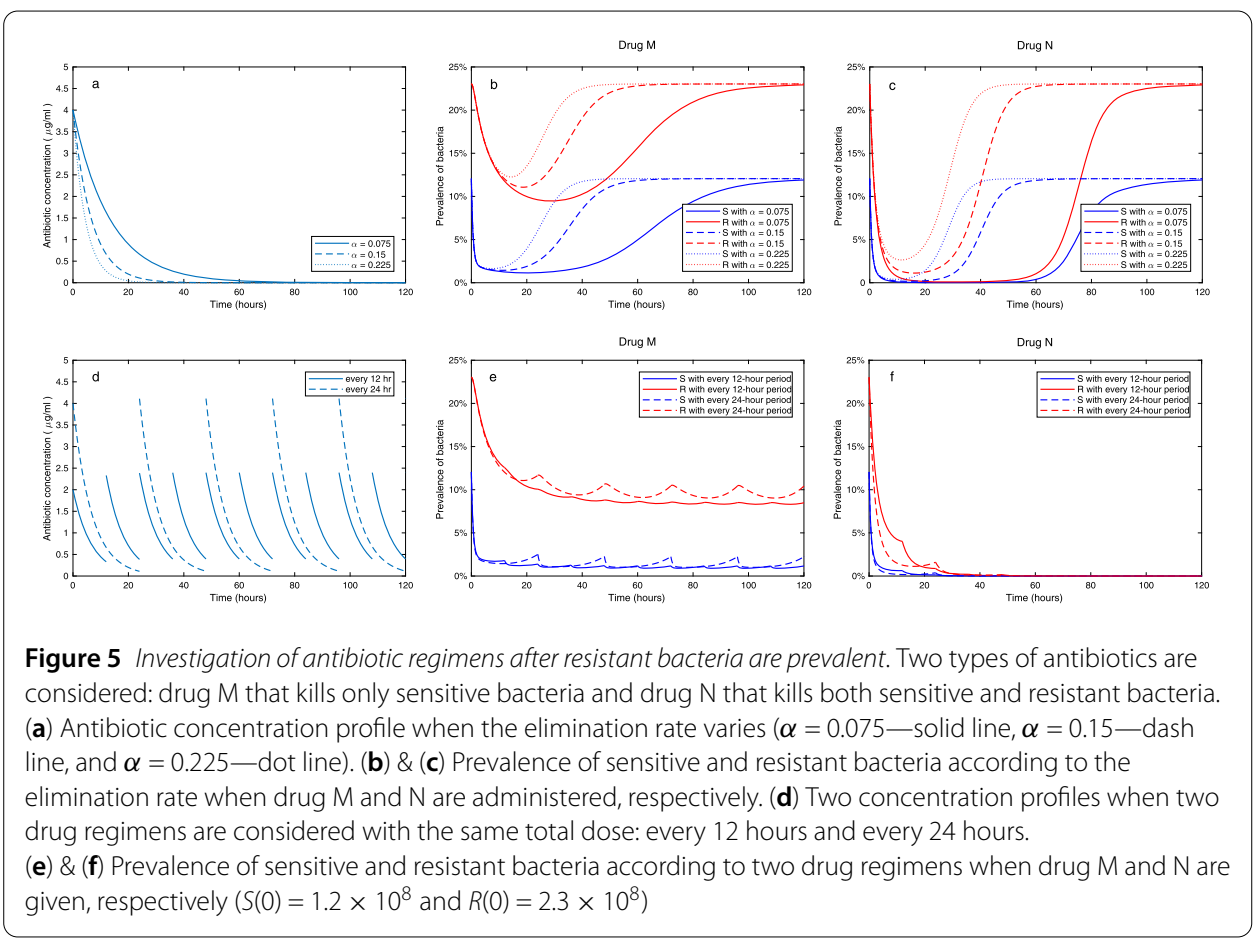

similar way with the antibiotic agent $M$, the prevalence of sensitive and resistant bacteria slowly increases when the elimination rate is small. Because the therapeutic agent is usually administered in multiple doses within a certain length of time, we further investigate this scenario of drug administration in Fig. 5(d)-(f). In Fig. 5(d), we demonstrate two strategies of drug administration for treating a patient in five days: every 12 hours and every 24 hours. Note that the total amount of drug in the two strategies is equivalent. For the antibiotic agent M, both populations of bacteria are present (see Fig. 5(e)). Moreover, with the same amount of drug administration, the prevalence of bacteria is lower when the antimicrobial agent is frequently administered; however, results are not significantly different. Figure 5(f) suggests that when the antimicrobial agent $\mathrm{N}$ that can kill both bacteria is applied, both bacteria die out within the treatment period. In this strategy, as the bacteria population dies out quickly within 20 hours, with a lower dose of the every 12hour strategy the sensitive and resistant bacteria are more prevalent than those resulting from the every 24-hour strategy.

To explore the time-delay effects in drug administration on the prevalence of sensitive and resistant bacteria, two delay schemes are introduced. The first scheme is for investigating the effects of a delay in administering the initial dose of an antibiotic agent. The drug concentration according to time is shown in Fig. 6(a). The initial dose is assumed to be given with no delay, delay for two days, or delay for four days. The second scheme is for investigating the effects of a delay in administering the second dose. The second dose is assumed to be given after 24 hours, after 36 hours, and after 48 hours. When the delay is present in administration of the initial dose, both bacterial populations only start decreasing right after the administration following the drug effects and then increase again after the drug wanes off. Similar trends occur for both types of drugs. We further investigate the effect of a delay by using the area under a curve (AUC) of each bacterial population that reflects its prevalence as shown in Table 2. As a result, the higher value of AUC suggests 


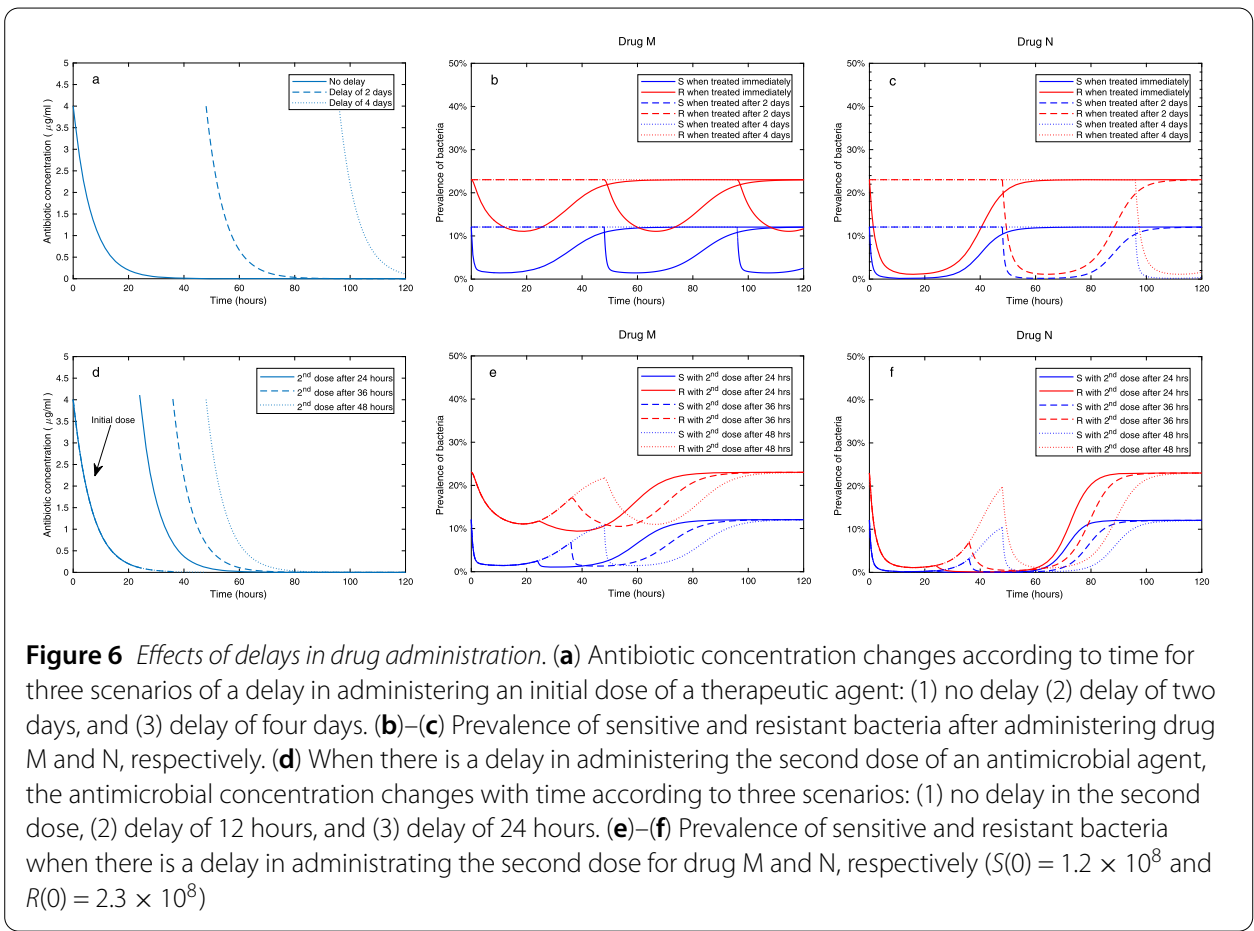

Table 2 Areas under the prevalence curves according to the time period of 8 and 12 days for sensitive and resistant bacteria

\begin{tabular}{|c|c|c|c|c|c|}
\hline \multirow[t]{2}{*}{ Delay $\tau$} & & \multicolumn{2}{|l|}{ Drug M } & \multicolumn{2}{|l|}{ Drug N } \\
\hline & & Sensitive & Resistant & Sensitive & Resistant \\
\hline$\tau=0$ & $\begin{array}{l}8 \text { days } \\
12 \text { days }\end{array}$ & $\begin{array}{r}2.661829 \\
12.830115\end{array}$ & $\begin{array}{l}19.462309 \\
39.866851\end{array}$ & $\begin{array}{l}0.175281 \\
3.860024\end{array}$ & $\begin{array}{l}0.841777 \\
7.259887\end{array}$ \\
\hline$\tau=2$ & $\begin{array}{l}8 \text { days } \\
12 \text { days }\end{array}$ & $\begin{array}{r}7.830120 \\
12.832599\end{array}$ & $\begin{array}{l}25.939458 \\
39.869256\end{array}$ & $\begin{array}{l}5.963989 \\
5.965214\end{array}$ & $\begin{array}{l}11.896001 \\
11.897438\end{array}$ \\
\hline$\tau=4$ & $\begin{array}{l}8 \text { days } \\
12 \text { days }\end{array}$ & $\begin{array}{l}12.998323 \\
14.239244\end{array}$ & $\begin{array}{l}32.415881 \\
41.570756\end{array}$ & $\begin{array}{l}11.752682 \\
11.752696\end{array}$ & $\begin{array}{l}22.950139 \\
22.950224\end{array}$ \\
\hline
\end{tabular}

higher prevalence of bacteria. In Table 2, we study the AUCs of sensitive and resistant bacteria within two time periods, 8 and 12 days. Based on our results, having a delay may generally lead to higher prevalence of bacteria for both types of antibiotics. For the antibiotic agent $M$, when the observation days increase from 8 days to 12 days, the number of bacteria increases. However, the difference of bacterial numbers between 8 and 12 days shows a declining trend when the delay of drug administration increases. For the antibiotic agent $\mathrm{N}$, such difference is only present when there is no delay in administration. Moreover, in this type of drug sensitive and resistant bacteria are not significantly different in numbers when the delay increases for both periods of observation days.

Furthermore, we investigate how the switching of antibiotic agents with different spectra of activity takes effect on the prevalence of bacteria in Fig. 7. As using only the single antibiotic agent $M$ leads to higher prevalence of both bacteria, Fig. 7(a)-(c) demonstrates that later introduction of drug $\mathrm{N}$ in the treatment course started with the antibiotic agent $M$ leads to the reduction of prevalence of bacteria in comparison to the treatment course with the single drug $\mathrm{M}$. The earlier the administration of $\mathrm{N}$, the lesser the prevalence of bacteria. Next, we explore a scenario that the antibiotic agent $\mathrm{M}$ is administered following 

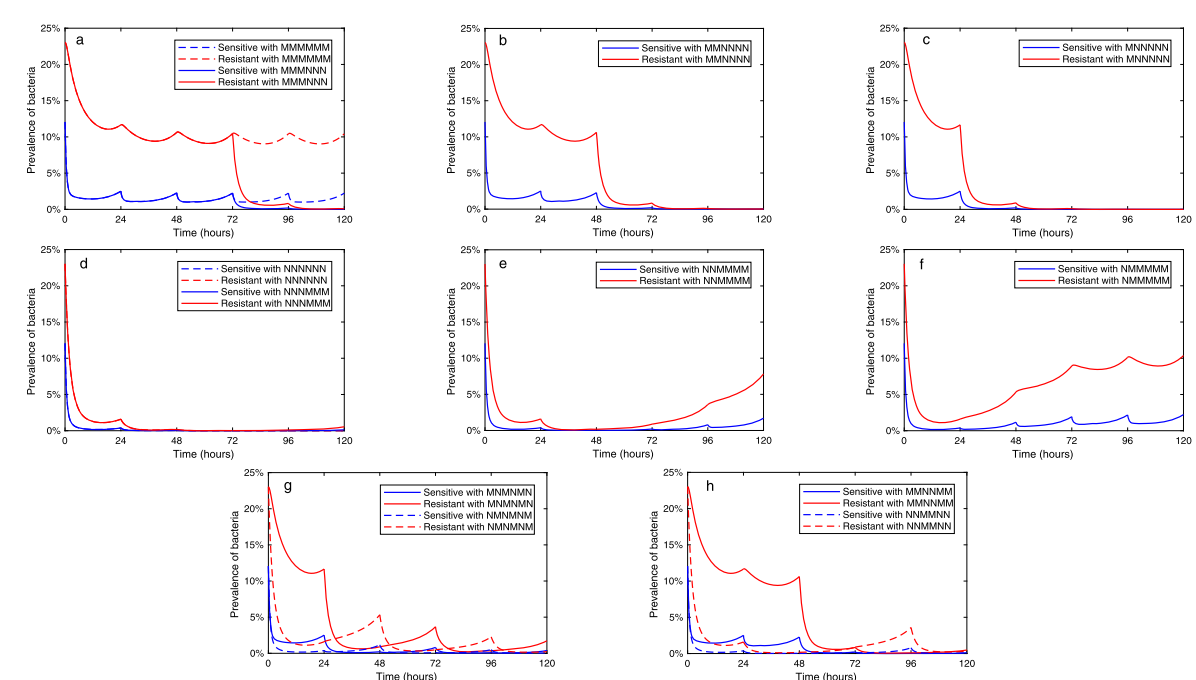

Figure 7 Switching of antibiotic agents. (a) Prevalence of sensitive and resistant bacteria with exposure to the antibiotic M: without and with introduction of the antibiotic $\mathrm{N}$ (dashed line for administration of the antibiotic $M$ only and solid line for administration of three doses of the antibiotic $M$ followed by three doses of the antibiotic N. (b) \& (c) Prevalence of sensitive and resistant bacteria with administration of two doses of the antibiotic $\mathrm{M}$ and four doses of the antibiotic $\mathrm{N}$ and with administration of one dose of the antibiotic $\mathrm{M}$ and four doses of the antibiotic N, respectively. (d) Prevalence of sensitive and resistant bacteria with exposure to the antibiotic $\mathrm{N}$ : without and with introduction of the antibiotic $\mathrm{M}$ (dashed line for administration of the antibiotic $\mathrm{N}$ only and solid line for administration of three doses of the antibiotic $\mathrm{N}$ followed by three doses of the antibiotic M. (e) \& (f) Prevalence of sensitive and resistant bacteria with administration of two doses of the antibiotic $\mathrm{N}$ and four doses of the antibiotic $\mathrm{M}$ and with administration of one dose of the antibiotic $\mathrm{N}$ and four doses of the antibiotic $\mathrm{M}$, respectively. ( $\mathbf{g}$ ) Prevalence of sensitive and resistant bacteria according to antibiotic switching between $\mathrm{M}$ and $\mathrm{N}$ starting with $\mathrm{M}$ (solid line) and $\mathrm{N}$ (dashed line). (h) Prevalence of sensitive and resistant bacteria switched between two doses of $\mathrm{M}$ and two doses of $\mathrm{N}$ starting with with $\mathrm{M}$ (solid line) and $\mathrm{N}$ (dashed line). $\left(S(0)=1.2 \times 10^{8}\right.$ and $R(0)=2.3 \times 10^{8}$ )

the prior treatment by the antibiotic agent $\mathrm{N}$ (see Fig. 7(d)-(f)). We find that increasing the administration of M may lead to higher prevalence of bacteria. In Fig. 7(g) \& (h), when antibiotics are switched between two agents, our results suggest that starting a treatment course with $\mathrm{N}$ may lead to the lower prevalence, and the one-dose switching style of antibiotics results in a better outcome for the treatment than the two-dose switching style.

As bacteria could have different characteristics, we study how different conjugation rate and probability of losing a plasmid affect the treatment strategies on the prevalence of bacteria. Figure 8(a)-(f) highlights the different outcomes following different values of $\lambda$ and $p$ after drug administration. Figure 8(a) shows that when a single agent is used in the treatment course, it provides a better outcome when bacteria have a tendency of losing a plasmid and the conjugation rate is high. Similarly, for other switching scenarios of treatment, resistant bacteria are significantly reduced when bacteria have the higher probability of losing a plasmid and the higher conjugation rate. The results correspond to the results illustrated in Fig. 3(d) \& (e) (see dashed lines) that by fixing the resistance acquisition number and varying both the conjugation rate and the probability of losing a plasmid, it is possible that lower values of $\lambda$ and $p$ result in higher prevalence of resistant bacteria during the treatment therapy. In addition, for all the treatment scenarios, bacteria are not significantly reduced when they are less likely to lose a plasmid and the conjugation rate is low. 

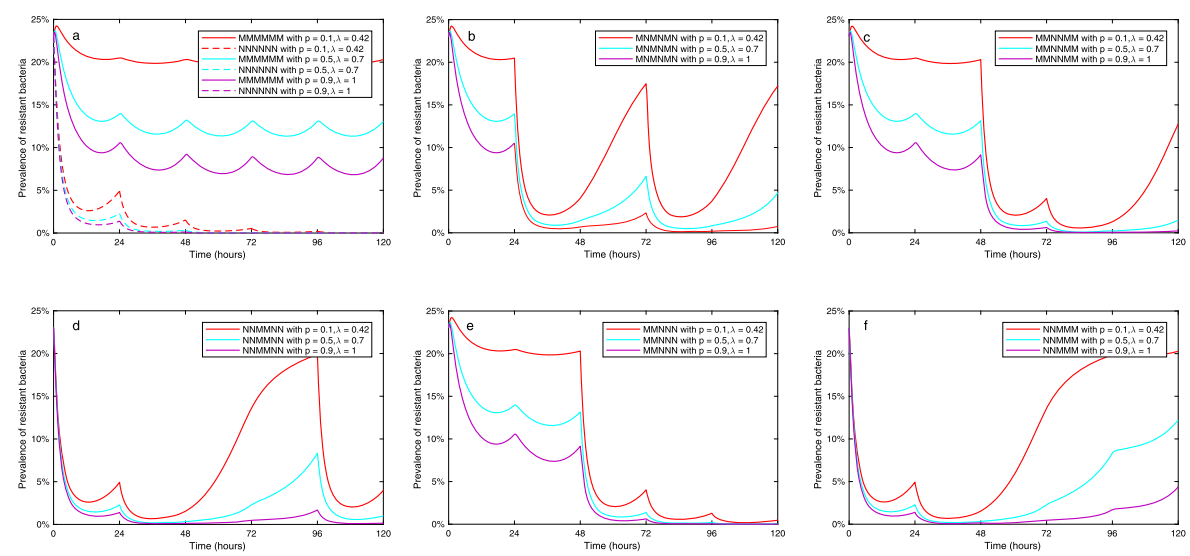

Figure 8 Characteristic of resistant bacteria. Three different characteristics of bacteria by fixing the resistance acquisition number equaling 3 are considered: (1) strain with less likelihood of losing a plasmid and horizontally transferring a plasmid $p=0.1, \lambda=0.65$ (red line), (2) strain with intermediate likelihood of loosing a plasmid and horizontally transferring a plasmid $p=0.5, \lambda=1.05$ (cyan line), and (3) strain with more likelihood of losing a plasmid and horizontally transferring a plasmid $p=0.9, \lambda=1.45$-purple line. (a) Prevalence of sensitive and resistant bacteria when the antibiotic M is administered only (solid line) and when the antibiotic $\mathrm{N}$ is administered only (dashed line). (b) Prevalence of sensitive and resistant bacteria for the one-dose antibiotic switching of M and N. (c) \& (d) Prevalence of sensitive and resistant bacteria for two-dose antibiotic switching of $\mathrm{M}$ and $\mathrm{N}$ starting with $\mathrm{M}$ and $\mathrm{N}$, respectively. (e) Prevalence of sensitive and resistant bacteria with two doses of $\mathrm{M}$ and three doses of $\mathrm{N}$. ( $\mathbf{f}$ ) Prevalence of sensitive and resistant bacteria with two doses of $\mathrm{N}$ and three doses of $\mathrm{M} .\left(\mathrm{S}(0)=1.2 \times 10^{8}\right.$ and $\left.R(0)=2.3 \times 10^{8}\right)$

\section{Discussion}

The ability of bacteria to acquire resistance to antibiotics has proven to have a dramatic impact on patient therapy [10]. One important process to acquire resistance that DNA material is transferred between bacterial cells by cell-to-cell contact is bacterial conjugation. The conjugation has been recognized as an important process responsible for the spread of resistant genes between patients under sublethally antibiotic treatment in the hospital environment. In this study, we develop a mathematical model that takes into account bacterial conjugation and drug effects to investigate the presence of sensitive and resistant bacteria in a human host. We also explore the effects of model parameters such as drug efficacy, conjugation rate, and the probability of losing a plasmid. Drug regimens, delay in administering a therapeutic agent, and combination of antibiotic agents are also investigated.

As resistant bacteria are allowed to lose plasmids, the sensitive bacteria are always present in the system. In this study, the resistance acquisition number $\left(R_{0}\right)$ that helps determine whether resistant bacteria can coexist with sensitive bacteria is calculated. We found that it depends on the ability of bacteria to transfer genes or the bacterial conjugation rate, the bacterial growth rate, the death rate of bacteria, and the probability of losing a plasmid. If $R_{0}<1$, only sensitive bacteria persist in the bacterial population. When $R_{0}>1$, both sensitive and resistant bacteria coexist within a host. These results correspond to our numerical results. Our study suggests that certain parameters affect the prevalence of sensitive and resistant bacteria. When the conjugation rate increases, the prevalence of sensitive bacteria declines while the prevalence of resistant bacteria increases. This is opposite to when the probability of losing a plasmid increases. In such a case, sensitive bacteria are more prevalent while resistant bacteria are more likely to lose their plasmids. 
In comparison to the probability of losing a plasmid, the conjugation rate has a greater impact on prevalence of sensitive and resistant bacteria when it is large enough. Note that the resistance acquisition number may be useful in identifying the parameters that may affect the dynamics of resistant bacteria, and it can be further investigated by a sensitivity analysis technique to determine which parameters have more impact on the prevalence of resistant bacteria.

Previous studies have demonstrated that pharmacodynamic parameters play an important role in the emergence of resistance [24, 25,27]. Here, we explore the effect of drug efficacy on the prevalence of sensitive and resistant bacteria by studying the ratio between the maximum kill rate of resistant bacteria and the maximum kill rate of sensitive bacteria. The reduced ability to kill resistant bacteria over sensitive bacteria more dramatically affects the prevalence of resistant bacteria than the prevalence of sensitive bacteria. In addition, our findings suggest that increasing the killing ability of antibiotic on resistant bacteria may not significantly reduce the presence of sensitive bacteria. Our results also suggest that antibiotic that lasts longer in the body is more likely to help reduce the prevalence of both bacteria. However, in reality the longer presence of antibiotic may relatively lead to accumulation of it in the body and result in toxic side effects [32]. Generally, antibiotic may be eliminated by being converted to metabolites or eliminated in feces or urine. Drug concentration and the dosing interval have been recognized as important factors that optimize therapeutic outcomes and minimize toxicity. Our findings correspond with previous findings suggesting multiple doses of antibiotics [22, 23]. Additionally, we investigate how two drug regimens with two dosing intervals and dosages affect the prevalence of sensitive and resistant bacteria for two types of drugs with different properties, drug $\mathrm{M}$ and $\mathrm{N}$. The results highlight that for drug $\mathrm{M}$ that kills only sensitive bacteria larger dosage and longer dosing interval may result in higher prevalence of resistant bacteria. This result is in agreement with a preceding study suggesting moderate treatments with appropriate timing, reduced drug dosage, and short treatment duration to limit the pathogen burden and selection of resistance [17]. However, our results also suggest that the larger dose and longer interval may positively lead to better outcomes for drug $\mathrm{N}$ that kill both bacteria.

Preceding studies suggest that delayed treatment may cause therapeutic failures and mortality of patients [40,41]. Hence, it is important to understand the effects of delays of antibiotic administration to minimize those consequences. To explore the effects of delay in this study, we consider two types of delays in administration of drug $\mathrm{M}$ and drug $\mathrm{N}$ : delay in administering an initial dose and delay in administering a second dose. Our results predict the negative impact of both types of delays on the prevalence of sensitive and resistant bacteria. The longer the delay lasts, the higher the prevalence of bacteria is. This is in agreement with the modeling study by D'Agata et al. [19] and the preceding empirical studies promoting the early administration of antibiotics to prevent treatment failures $[40,41]$. Moreover, our results also suggest that the delays may have a greater impact on the prevalence of resistant bacteria in comparison to sensitive bacteria when drug $\mathrm{N}$ is administered. They may lead to the increasing number of resistant bacteria. In contrast, the findings here contradict with a previous study when host immunity is taken into account [17].

Based on our results, a treatment course with the single antibiotic $\mathrm{N}$ is most effective while a treatment course with the single antibiotic $M$ is less effective. As antibiotics could have toxicity or other side effects, in this work, we investigate an impact of antibiotic 
switching and how switching between two antibiotics with different spectra ( $M$ which is less powerful and $\mathrm{N}$ which is more powerful) would affect the prevalence of bacteria. We found that introduction of the antibiotic $\mathrm{N}$ into the prior treatment with $\mathrm{M}$ could help reduce the prevalence of bacteria while introduction of the antibiotic $M$ into the prior treatment with $\mathrm{N}$ could lead to the higher prevalence of bacteria later. Our results are in agreement with empirical studies supporting the use of a more powerful antibiotic agent for a whole treatment course or the use of the powerful antibiotic after another antibiotic agent for better treatment outcomes [42, 43]. However, the results are in contrast with some preceding studies suggesting that switching from a less powerful treatment to a more powerful antibiotic agent could lead to better outcomes than a scenario that only a more powerful antibiotic agent is administered [44]. Our findings also suggest that switching the antibiotics by starting with the antibiotic $\mathrm{N}$ results in better treatment outcomes than starting with the antibiotic M. In addition, we explore how characteristics of bacteria affect the prevalence of bacteria. Our results suggest that treatment is more effective for bacteria with the higher probability of losing a plasmid and the higher conjugation rate. Our results are in agreement with some preceding studies that highlighted the importance of interval between treatments to plasmid-borne antibiotic resistance [45].

All in all, we believe that our study helps gain a better understanding into the population dynamics of sensitive and resistant bacteria under a therapeutic treatment, the effects of certain factors involving bacterial mechanisms, and other effects such as drug regimens, drug efficacy, delays in administrations, antibiotic switching, and characteristics of bacteria strains. As changing certain parameters may affect the overall results, one limitation of this study is related to some specific values of parameters. In each numerical investigation, certain parameters related to possible scenarios are altered to specifically investigate their effects on the presence of sensitive and resistance bacteria while some other parameters remain fixed and are subject to their realistic values as possible. We believe that our study helps illustrate the usefulness of mathematical models to investigate treatment strategies, and the work can be extended to explore other parameters and drug regimens to optimize the prevalence of resistant bacteria.

\section{Acknowledgements}

Both authors would like to thank Prof. Prasit Palittapongarnpim for useful suggestions.

Funding

PT and FC would like to thank the Research Fund for DPST Graduate with First Placement (02/2557) for the equipment, software support, and publication fee. The funding body had no role in the study design, analysis and interpretation of results nor in writing the manuscript.

\section{Abbreviations}

ARB, Antimicrobial-resistant bacteria; HGT, Horizontal gene transfer; MRSA, Methicillin-resistant Staphylococcus aureus; DNA, Deoxyribonucleic acid; AUC, Area under the curve.

Availability of data and materials

Not applicable.

Competing interests

The authors declare that they have no competing interests.

Authors' contributions

FC designed the study. Both developed the model and interpreted the results. PT performed the analytic calculations and the numerical simulations. Both wrote the manuscript, read and approved the final manuscript.

\section{Publisher's Note}

Springer Nature remains neutral with regard to jurisdictional claims in published maps and institutional affiliations. 
Received: 12 June 2020 Accepted: 11 May 2021 Published online: 12 June 2021

\section{References}

1. Llor, C., Bjerrum, L.: Antimicrobial resistance: risk associated with antibiotic overuse and initiatives to reduce the problem. Ther. Adv. Drug Saf. 5(6), 229-241 (2014)

2. Ventola, C.L.: The antibiotic resistance crisis: part 1: causes and threats. Pharm. Ther. 40(4), 277-283 (2015)

3. Mulvey, M.R., Simor, A.E.: Antimicrobial resistance in hospitals: how concerned should we be? Can. Med. Assoc. J. 20(4), 243-252 (2015)

4. Fletcher, S.: Understanding the contribution of environmental factors in the spread of antimicrobial resistance. Environ. Health Prev. Med. 180(4), 408-415 (2009)

5. Aslam, B., Wang, W., Arshad, M.l., et al.: Antibiotic resistance: a rundown of a global crisis. Infect. Drug Resist. 11, 1645-1658 (2018)

6. Blair, J.M.A., Webber, M.A., Baylay, A.J., et al.: Molecular mechanisms of antibiotic resistance. Nat. Rev. Microbiol. 13 42-51 (2015)

7. Raz, Y., Tannenbaum, E.: The influence of horizontal gene transfer on the mean fitness of unicellular populations in static environments. Genetics 185(1), 327-337 (2010)

8. Sommer, M.O.A., Munchk, C., Toft-Kehler, R.V.: Prediction of antibiotic resistance: time for a new preclinical paradigm? Nat. Rev. Microbiol. 15, 689-696 (2017)

9. Thomas, C.M., Nielsen, K.M.: Mechanisms of, and barriers to, horizontal gene transfer between bacteria. Nat. Rev. Microbiol. 3, 711-721 (2005)

10. Munita, J.M., Arias, C.A.: Mechanisms of antibiotic resistance. Microbiol. Spectr. 87, 1-37 (2016)

11. Richardson, L.A.: Understanding and overcoming antibiotic resistance. PLoS Biol. 15(8), 1-5 (2017)

12. Nair, C.G., Chao, C., Ryall, B., Williams, H.D.: Sub-lethal concentrations of antibiotics increase mutation frequency in the cystic fibrosis pathogen Pseudomonas aeruginosa. Lett. Appl. Microbiol. 56(2), 149-154 (2012)

13. Sengupta, S., Chattopadhyay, M.K., Grossart, H.-P.: The multifaceted roles of antibiotics and antibiotic resistance in nature. Front. Microbiol. 4(47), 1-13 (2013)

14. Huddleston, J.R.: Horizontal gene transfer in the human gastrointestinal tract: potential spread of antibiotic resistance genes. Infect. Drug Resist. 7, 167-176 (2014)

15. von Wintersdorff, C.J.H., Penders, J., van Niekerk, J.M., et al.: Dissemination of antimicrobial resistance in microbial ecosystems through horizontal gene transfer. Front. Microbiol. 7, 173 (2016)

16. Handel, A., Margolis, E., Levin, B.R.: Exploring the role of the immune response in preventing antibiotic resistance. J. Theor. Biol. 256, 655-662 (2009)

17. Gjini, E., Brito, P.H.: Integrating antimicrobial therapy with host immunity to fight drug-resistant infections: classical vs. adaptive treatment. PLoS Comput. Biol. 12, 1-34 (2016)

18. Ternent, L., Dyson, R.J., Krachler, A.-M., Jabbari, S.: Bacterial fitness shapes the population dynamics of antibiotic-resistant and susceptible bacteria in a model of combined antibiotic and anti-virulence treatment. J. Theor. Biol. 372, 1-11 (2015)

19. D'Agata, E.M.C., Durpont-Rounzeyrol, M., Magal, P., et al.: The impact of different antibiotic regimens on the emergence of antimicrobial-resistant bacteria. PLOS ONE 3(12), 1-10 (2009)

20. Beardmore, R.E., Peña-Miller, R.: Rotating antibiotics selects optimally against antibiotic resistance, in theory. Math. Biosci. Eng. 7, 527-552 (2010)

21. Torella, J.P., Chait, R., Kishony, R.: Optimal drug synergy in antimicrobial treatments. PLoS Comput. Biol. 6(6), 1-9 (2010)

22. Cogan, N.G., Brown, J., Darres, K., Petty, K.: Optimal control strategies for disinfection of bacterial populations with persister and susceptible dynamics. Antimicrob. Agents Chemother. 56, 4816-4826 (2012)

23. Paterson, L.K., Hoyle, A., Ochoa, G., et al.: Optimising antibiotic usage to treat bacterial infections. Sci. Rep. 6, 37853 (2016)

24. Regoes, R.R., Wiuff, C., Zappala, R.M., et al.: Pharmacodynamic functions: a multiparameter approach to the design of antibiotic treatment regimens. Antimicrob. Agents Chemother. 48, 3670-3676 (2004)

25. Nielsen, E.I., Cars, O., Friberg, L.E.: Pharmacokinetic/pharmacodynamic (PK/PD) indices of antibiotics predicted by a semimechanistic PKPD model: a step toward model-based dose optimization. Antimicrob. Agents Chemother. 55 , 4619-4630 (2011)

26. Opatowski, L., Guillemot, D., Boëlle, P.Y., Temime, L.: Contribution of mathematical modeling to the fight against bacterial antibiotic resistance. Curr. Opin. Infect. Dis. 24, 279-287 (2011)

27. Ankomah, P., Johnson, P.J., Levin, R.B.: The pharmaco -, population and evolutionary dynamics of multi-drug therapy: experiments with s. aureus and e. coli and computer simulations. PLoS Pathog. 9, 1-14 (2013)

28. Greenfield, B.K., Shaked, S., Marrs, C.F., et al.: Modeling the emergence of antibiotic resistance in the environment: an analytical solution for the minimum selection concentration. Antimicrob. Agents Chemother. 62, 1-16 (2018)

29. Champion, J.J., McNamara, P.J., Evans, M.E.: Pharmacodynamic modeling of ciprofloxacin resistance in Staphylococcus aureus. Antimicrob. Agents Chemother. 49, 209-219 (2005)

30. Czock, D., Keller, F.: Mechanism-based pharmacokinetic-pharmacodynamic modeling of antimicrobial drug effects. J. Pharmacokinet. Pharmacodyn. 34, 727-751 (2007)

31. Chung, P., McNamara, P.J., Champion, J.J., Evans, M.E.: Mechanism-based pharmacodynamic models of fluoroquinolone resistance in Staphylococcus aureus. Antimicrob. Agents Chemother. 50(9), 2957-2965 (2006)

32. Levison, M.E., Levison, J.H.: Pharmacokinetics and pharmacodynamics of antibacterial agents. Infect. Dis. Clin. North Am. 23(4), 791-815 (2009)

33. Gostelow, M., Gonzalez, D., Smith, P.B., Cohen-Wolkowiez, M.: Pharmacokinetics and safety of recently approved drugs used to treat methicillin-resistant Staphylococcus aureus infections in infants, children, and adults. Expert Rev. Clin. Pharmacol. 7(3), 327-340 (2014)

34. Ibargüen-Mondragón, E., Mosquera, S., Cerónand, M., et al.: Mathematical modeling on bacterial resistance to multiple antibiotics caused by spontaneous mutations. Biosystems 117(12), 60-67 (2014)

35. Haaber, J., Penades, J.R., Ingmer, H.: Transfer of antibiotic resistance in Staphylococcus aureus. Trends Microbiol. 25(11), 327-337 (2017) 
36. Nielsen, E.I., Friberg, L.E.: Pharmacokinetic-pharmacodynamic modeling of antibacterial drugs. Pharmacol. Rev. 65(3), 1053-1090 (2013)

37. Driessche, P.V.D., Watmough, J.: Reproduction numbers and sub-threshold endemic equilibria for compartmental models of disease transmission. Math. Biosci. 180, 29-48 (2002)

38. Grundmann, H., Hori, S., Winter, B., et al.: Risk factors for the transmission of methicillin-resistant Staphylococcus aureus in an adult intensive care unit: fitting a model to the data. J. Infect. Dis. 185, 481-488 (2002)

39. Spicknall, I.H., Foxman, B., Marrs, C.F., Eisenberg, J.N.S.: A modeling framework for the evolution and spread of antibiotic resistance: literature review and model categorization. Am. J. Epidemiol. 178(4), 508-520 (2013)

40. Proulx, N., Fréchette, D., Toye, B., et al.: Delays in the administration of antibiotics are associated with mortality from adult acute bacterial meningitis. Int. J. Med. Educ. 98(4), 291-298 (2005)

41. Mok, K., Christian, M.D., Nelson, S., Burry, L.: Time to administration of antibiotics among inpatients with severe sepsis or septic shock. Can. J. Hosp. Pharm. 67(3), 213-219 (2014)

42. de Jong, L.A.W., van der Linden, P.D., Roukens, M.M.B., et al.: Consecutive antibiotic use in the outpatient setting: an extensive, longitudinal descriptive analysis of antibiotic dispensing data in the Netherlands. BMC Infect. Dis. 19(1), 84 (2019)

43. Schweizer, M.L., Richardson, K., Sarrazin, M.S.V., et al.: Comparative effectiveness of switching to daptomycin versus remaining on vancomycin among patients with methicillin-resistant Staphylococcus aureus (MRSA) bloodstream infections. Clin. Infect. Dis. 72(Supplement 1), S68-S73 (2021)

44. Culshaw, D., Lamp, K.C., Yoon, M.J., Lodise, T.P.: Duration of prior vancomycin therapy and subsequent daptomycin treatment outcomes in methicillin-resistant Staphylococcus aureus bacteremia. Diagn. Microbiol. Infect. Dis. 83(2), 193-197 (2015)

45. Svara, F., Rankin, D.J.: The evolution of plasmid-carried antibiotic resistance. BMC Evol. Biol. 11(1), 130 (2011)

\section{Submit your manuscript to a SpringerOpen ${ }^{\circ}$ journal and benefit from:}

- Convenient online submission

- Rigorous peer review

- Open access: articles freely available online

- High visibility within the field

- Retaining the copyright to your article

Submit your next manuscript at $\boldsymbol{\nabla}$ springeropen.com 